\title{
In Situ Measurement of Seeking Speed and Seeking Induced Head-Disk Interface Instability in Hard Disk Drives
}

\author{
Yu Wang, ${ }^{1,2}$ Xiongfei Wei, ${ }^{3}$ Yanyang $\mathrm{Zi}^{1},{ }^{1}$ and Kwok-Leung Tsui ${ }^{2}$ \\ ${ }^{1}$ School of Mechanical Engineering, State Key Laboratory for Manufacturing and Systems Engineering, Xian Jiaotong University, \\ Xian 710049, China \\ ${ }^{2}$ Department of Systems Engineering and Engineering Management, City University of Hong Kong, Hong Kong \\ ${ }^{3}$ SAE Technologies Development (Dongguan) Co. Ltd., Dongguan 523087, China
}

Correspondence should be addressed to Yu Wang; ywang95@mail.xjtu.edu.cn

Received 29 April 2015; Accepted 28 June 2015

Academic Editor: Chuan Li

Copyright (C) $2015 \mathrm{Yu}$ Wang et al. This is an open access article distributed under the Creative Commons Attribution License, which permits unrestricted use, distribution, and reproduction in any medium, provided the original work is properly cited.

\begin{abstract}
This paper investigated the instability of head-disk interface caused by the voice coil motor (VCM) end crashing the crash stop during the seeking of magnetic head. To make the whole process of that clear, an in situ measurement method based on maximum likelihood estimation and extended Kalman filter for seeking speed at component level was developed first and was then calibrated by a high speed camera. Given a crash between VCM end and crash stop that may be a consequence of the continuous increasing seeking speed, the seeking speed was carefully controlled by using our developed method to find a critical value that may induce vigorous head-disk interface instability. Acoustic emission sensor and laser Doppler vibrometer were used to capture the transient dynamic behaviors of magnetic head when the crash is happening. Damage analysis and mode identification were carried out to reveal the relationship between the damage patterns on disk surface and head dynamics. The results of this study are helpful to optimize the track seeking profile during the HDD operation, as well as the design of components such as head and head arm.
\end{abstract}

\section{Introduction}

The areal density of the hard disk drive (HDD) is expected to reach multiseveral $\mathrm{Tb} / \mathrm{in}^{2}$ in next few years $[1,2]$. At such high density, the data transfer needs to further speed up due to the competition pressure from the solid-state drive (SSD) and the requirement of users. The access time or response time of a rotating drive that measures the time of HDD components' response before the drive transferring data is mainly related to the mechanical nature of the rotating disks and moving heads. However, due to the limitations of mechanical components in HDDs, low-speed motion has become one of the chief obstacles for further increasing the speed of data transfer $[3,4]$. Track following and seeking is a key step in the loop of data transfer in HDDs, and thus increasing seeking speed is raised as a critical task in HDDs.

Generally, head seeking is implemented by inputting a digital current into the voice coil motor (VCM) to drive the head arm assembly move. To provide a specific speed, an available method is to add a constant current to VCM for a prescribed amount of time. However, this method may not achieve the precise speed control due to the disturbances, such as friction of the pivot bearings and the resistance force of data flex cable $[5,6]$. An alternative method taking advantage of back electromotive force (EMF) was proposed to create a negative acceleration in order to produce the VCM velocity which is widely adopted in current HDDs [7-9]. The mechanism of this method is that a back EMF representing the velocity of the VCM will be generated when the power is cut off. The seeking speed can be attained by adjusting the back EMF.

For a safety consideration, two crash stops were designed to limit the rotation of the VCM to prevent the head from moving off of the disk or hitting the spindle during head seeking [10]. No matter which manner used for VCM control, when the seeking speed is too high, it has a risk that the end of VCM may crash the crash stop. Such a crash may induce the HDD instability such as head slap on disk and head/disk damage. Although this topic is essential, there is rarely report on that in literature. It is therefore necessary to have a better 


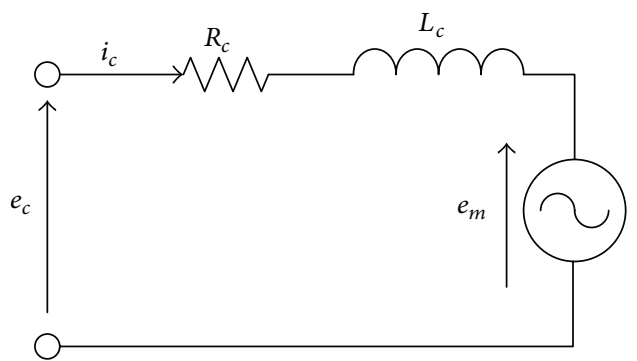

FIGURE 1: Simplified electronic circuit of a typical VCM.

understanding for the whole process of that, especially at component level, which is generally followed by system integration and thus is highly related to the component design and the yielding of the final products.

Given the above considerations, this paper investigated the instability of HDI caused by the VCM crashing the crash stop during head seeking at the component level. To investigate the effect of seeking speed on anti-impact performance of head-disk interface, a maximum likelihood estimation (MLE) and extended Kalman filter (EKF), MLE-EKF, method was developed to estimated and control the speed flexibly. The EKF method is an improved method of the Kalman filter that is incapable of handling the nonlinear problems $[11,12]$. Then, different speeds were carefully controlled to find a critical value that may induce HDI instability. Acoustic emission (AE) sensor and laser Doppler vibrometer (LDV) were used to capture the transient dynamic behaviors of head when the crash is happening. Damage analysis and mode identification were carried out to reveal the relationship between the damage patterns on disk surface and head dynamics.

\section{Design and Calibration of Seeking Speed}

2.1. Mathematic Model. Before implementing the experiment, the seeking speed of head should be well controlled and calibrated. To this end, a drive-based tester was used to implement the seeking test. Head seeking controlling method by the back EMF-based control technology applies the back EMF that is generated by coil movement in the magnetic field to drive the VCM to move. A simplified electronic circuit is shown in Figure 1, and the electrical dynamics of the VCM can be described as [13]

$$
L_{c} \frac{d i_{c}(t)}{d t}+R_{c} i_{c}(t)+e_{m}(t)=e_{c}(t)
$$

where $L_{c}$ is the inductance of coil, $R_{c}$ is the resistance of coil, $e_{c}$ is applied voltage, and $e_{m}$ is the induced back EMF in the coil due to the permanent magnets. According to Lenz's law, the running of VCM generates a back EMF proportional to rotational velocity. Therefore, the back EMF can be calculated by $e_{m}=K_{e} \omega$, and $K_{e}$ is the back EMF constant.

According to the mechanical dynamic model of the VCM, the current can be transferred to the rotation speed by $[14,15]$

$$
K_{t} i_{c}(t)-T_{f}-K_{c} \cdot x=J \dot{\omega}(t),
$$

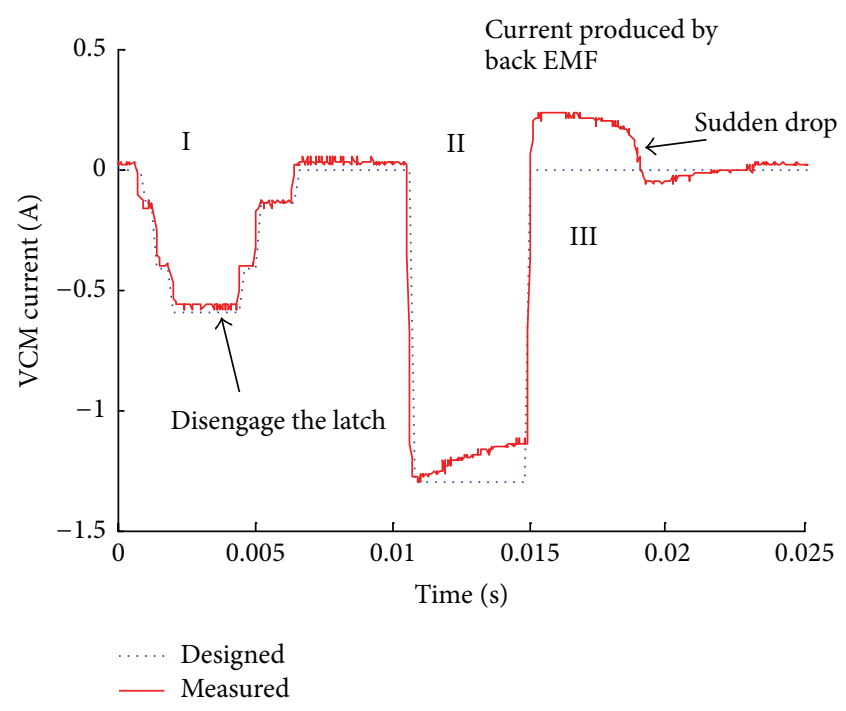

FIGURE 2: The measured VCM current profiles during seeking.

where $K_{t}$ is the torque constant, $i_{c}$ is the coil current, $J$ is the inertia of head arm, $T_{f}$ is the torque generated by pivot friction force, $K_{c}$ is the stiffness of flex cable, $x$ is the displacement, and $\omega$ is the angular velocity. The values of $K_{t}$ and $J$ used in this experiment are $0.026 \mathrm{Nm} / \mathrm{A}$ and $0.1309 \times$ $10^{-5} \mathrm{kgm}^{2}$, respectively. By integrating $\dot{\omega}$, the rotation speed of arm can be obtained. Given the arm length $l$, the head seeking speed can be calculated. It is therefore necessary to know the implemented current and estimate the key parameters including $K_{t}, T_{f}$, and $J$ before identifying the seeking speed.

A programmable power source was used to control the current of VCM so as to control the seeking speed. Figure 2 shows the designed waveform of VCM current and the measured current profile by an oscilloscope. The measured current profile consists of three phases. Phase I provided the power for VCM to disengage the latch. Phase II generated the seeking speed. Phase III was produced by the back EMF. It can be observed at phase III that a sudden drop occurred at $0.018 \mathrm{~s}$, implying that a crash happened. A more detailed explanation was given in Section 3. When the current turned off, the rotational speed of VCM can be directly determined by back EMF. Using this property, the unknown parameters $T_{f}$ and $K_{c}$ can be estimated accordingly.

2.2. Parameter Estimation. To estimate the parameters in (2), it is necessary to reconsider the mechanics of VCM. When the VCM current is cut off, (2) becomes

$$
T_{f}+K_{c} \cdot x=-J \dot{\omega}(t)
$$

assuming that the displacement of flex cable has a linear relationship with the duration

$$
x=a+b t .
$$


By integrating $\dot{\omega}(t)$, the angular speed of VCM can be calculated by

$$
\widehat{\omega}=\omega_{0}-\frac{1}{J}\left[\left(T_{f}+K_{c} a\right) t-\frac{1}{2} K_{c} b t^{2}\right] .
$$

For the sake of simplicity, (5) can be simplified by combining the parameters, that is, letting $C_{1}=(1 / J)\left[T_{f}+\right.$ $\left.K_{c} a\right], C_{2}=K_{c} b / J$, and (5) become

$$
\widehat{\omega}=\omega_{0}-C_{1} t-\frac{1}{2} C_{2} t^{2}
$$

Due to the disturbance, the measurement always companied with measurement error. The measurement error can be regarded as an independent and identically distributed normal random variable. Given the measurement error $\varepsilon \sim$ $N\left(0, \sigma^{2}\right)$, the angular speed can be written as

$$
\omega=\widehat{\omega}+\varepsilon .
$$

The angular speed follows $\omega \sim N\left(\widehat{\omega}, \sigma^{2}\right)$. To estimate the parameters $C_{1}, C_{2}$, and $\sigma$, the maximum likelihood estimation method was used, which then turns to the logarithmic likelihood function of the angular speed:

$$
\begin{aligned}
\log L & =\sum_{i=1}^{m}\left(\frac{n_{i}}{2}(-\ln (2 \pi)-2 \ln \sigma)\right. \\
- & \left.\frac{\sum_{k=1}^{n_{i}}\left(\omega_{i}-\omega_{0}+C_{1} t+(1 / 2) C_{2} t^{2}\right)^{2}}{2 \sigma^{2}}\right) .
\end{aligned}
$$

By maximizing the log-likelihood function, the parameters $C_{1}, C_{2}$, and $\sigma$ can be obtained.

Three VCM current waveforms were used to build the likelihood function, as illustrated in Figure 3. By maximizing the log-likelihood function (8), the estimated parameters are $C_{1}=245.9, C_{2}=0$, and $\sigma=0.54$. The seeking speed can be calculated by integrating $\dot{\omega}$ (when VCM current was supplied) or by $e_{m} / K_{e}$ (when VCM current was cut off).

2.3. Real-Time Speed Estimation. We treated these estimated parameters as the initial parameters for an EKF; a real-time VCM speed can be obtained through the prediction and update steps in the EKF. We assume the state of the EKF is $x=\left[\begin{array}{lll}C_{1} & C_{2} & \omega_{0}\end{array}\right]$. According to the mechanical dynamic model of the VCM, the state and measurement function of the EKF can be expressed as follows.

State model is

$$
\begin{aligned}
x_{k} & =f\left(x_{k-1 \mid k-1}, k-1\right)+q_{k-1}, \\
f\left(x_{k-1 \mid k-1}, k-1\right) & =\left[\begin{array}{lll}
1 & 0 & 0 \\
0 & 1 & 0 \\
0 & 0 & 1
\end{array}\right]\left[\begin{array}{l}
C_{1, k-1} \\
C_{2, k-1} \\
\omega_{0, k-1}
\end{array}\right],
\end{aligned}
$$

where $q_{k-1}=\left[\begin{array}{lll}v_{C_{1}} & v_{C_{2}} & v_{\omega_{0}}\end{array}\right], v_{C_{1}} \sim N\left(0, \sigma_{C_{1}}\right), v_{C_{2}} \sim N(0$, $\left.\sigma_{C_{2}}\right)$, and $v_{\omega_{0}} \sim N\left(0, \sigma_{\omega_{0}}\right)$.

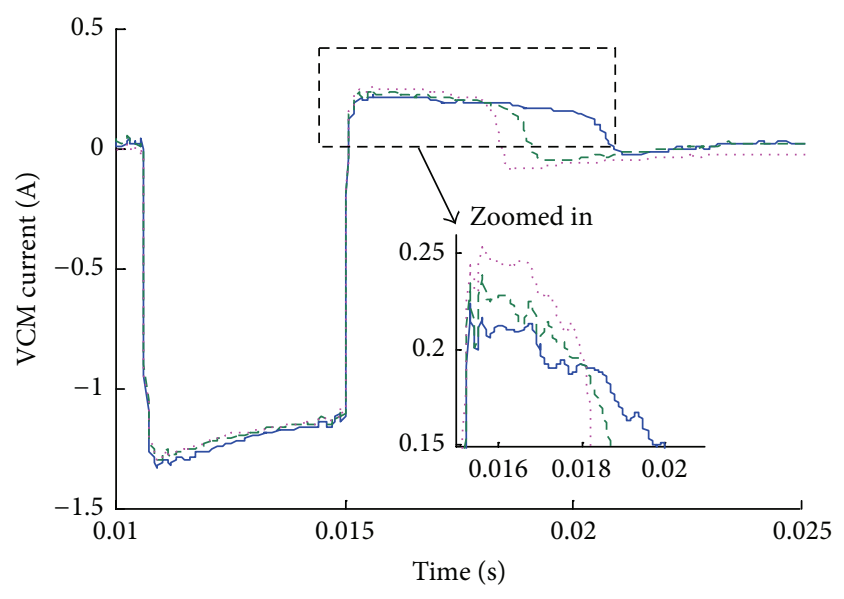

FIgURE 3: Three VCM current waveforms to generate different seeking speed.

Measurement model is

$$
\begin{aligned}
\omega_{k} & =h\left(x_{k}, k\right)+v, \\
h\left(x_{k}, k\right) & =\omega_{0, k-1}-C_{1, k} t-\frac{1}{2} C_{2, k} t^{2},
\end{aligned}
$$

where $v \sim N\left(0, \sigma_{y}\right)$.

Based on this state-space function, the first order EKF can be used to smooth the raw data. The steps for the first order EKF are as follows [12].

Predict

$$
\begin{aligned}
& x_{k \mid k-1}=f\left(x_{k-1 \mid k-1}, k-1\right), \\
& P_{k \mid k-1} \\
& \quad=F_{x}\left(x_{k-1 \mid k-1}, k-1\right) P_{k-1 \mid k-1} F_{x}^{T}\left(x_{k-1 \mid k-1}, k-1\right) \\
& \quad+Q_{k-1},
\end{aligned}
$$

where $F_{x}$ is defined to be $\partial f / \partial x$.

Update

$$
\begin{aligned}
v_{k \mid}= & \omega_{k}-h\left(x_{k}, k\right), \\
S_{k \mid} & \\
= & H_{x}\left(x_{k-1 \mid k-1}, k-1\right) P_{k-1 \mid k-1} H_{x}^{T}\left(x_{k-1 \mid k-1}, k-1\right) \\
& +R_{k}, \\
K_{k}= & P_{k \mid k-1} H_{x}^{T}\left(x_{k-1 \mid k-1}, k-1\right) S_{k}^{-1}, \\
x_{k \mid k}= & x_{k \mid k-1}+K_{k} v_{k \mid}, \\
P_{k \mid k}= & P_{k \mid k-1}-K_{k} S_{k \mid} K_{k}^{T},
\end{aligned}
$$

where $H_{x}$ is defined to be $\partial h / \partial x$.

To illustrate the effectiveness of the proposed method, the EMF induced speed was estimated by EKF. As shown in Figure 4, the estimated speed follows the measured speed well 


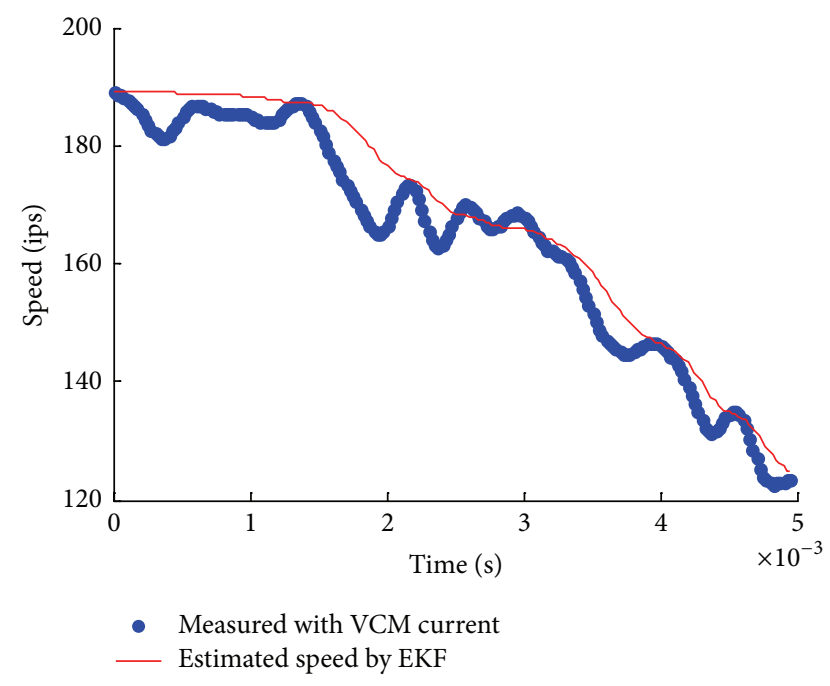

FIGURE 4: Real-time estimation of the head speed by EKF.

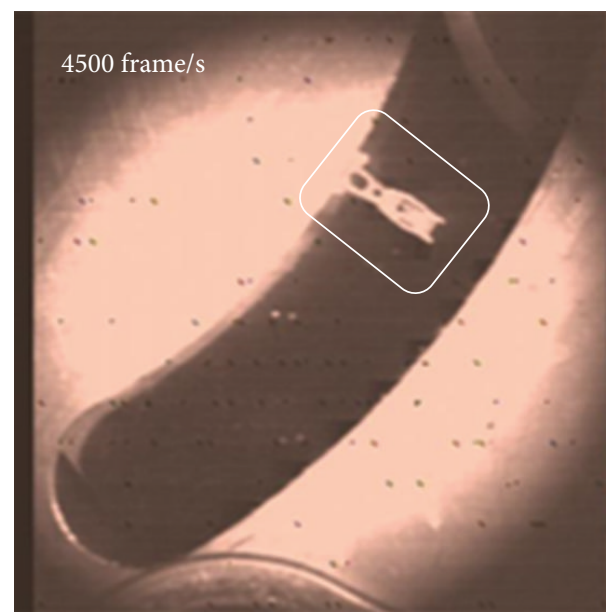

Figure 5: The pattern captured by high speed camera.

as the maximum relative error is $8 \%$. This indicates that our proposed speed estimation method is valid for estimating the head speed.

2.4. Speed Calibration by High Speed Camera. To verify the aforementioned calculated seeking speed, a high speed camera taking the snapshots for head arm with a speed of 4500 frames per second was used. The head arm pattern at any location, as shown in Figure 5, was continuously captured by the camera.

Using a National Instruments image identification software to measure the angular displacement $\Delta \theta$ of the featured pattern of head arm at a small time interval $\Delta t$ during seeking process, the speed can be calculated by $\Delta \theta / \Delta t$.

A correlation between our estimated speed and the measured speed by camera was conducted, as illustrated by Figure 6. As observed from this figure, a good correlation exists between the estimated and the measured results as

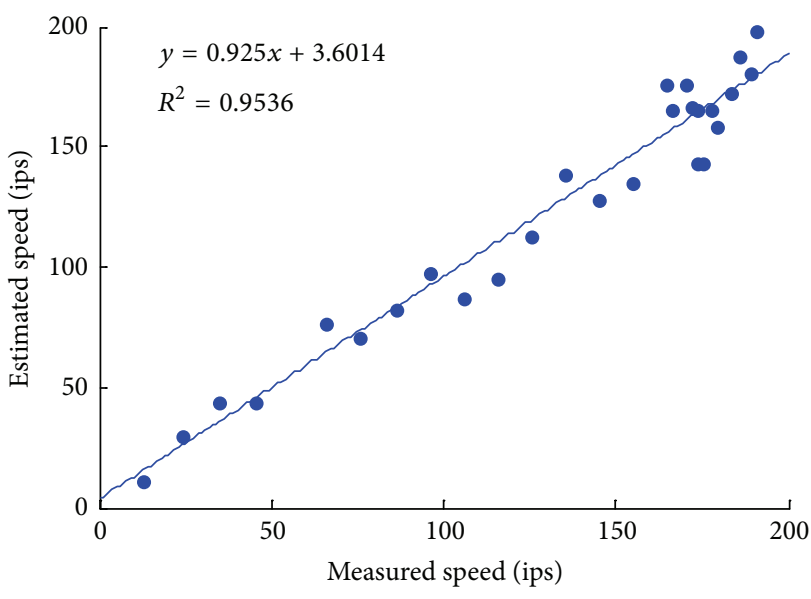

FIGURE 6: The seeking speed calibrated by high speed camera.

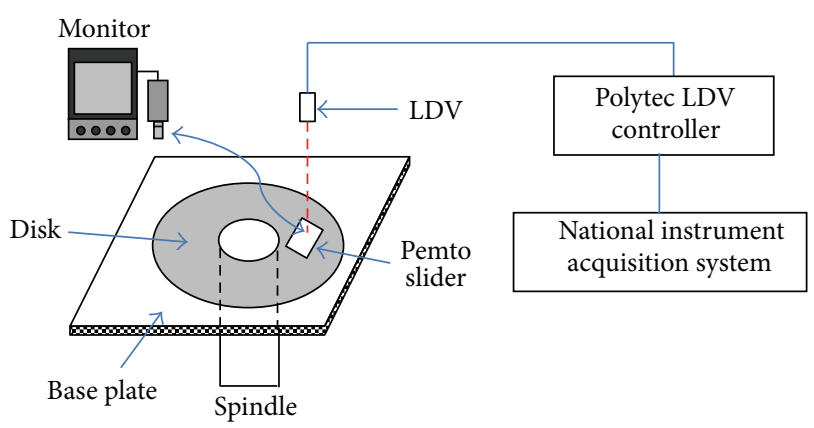

FIgURE 7: The schematic of the experiment setup.

$R^{2}$ is approximately 0.95 . This means the seeking speed can be well quantified by our estimated speed.

\section{Experimental Setup for Crashing Characterization}

Based on back EMF control technology, a component-level evaluation system for seeking was developed. The schematic of the experimental configuration is shown in Figure 7. An acoustic emission sensor was mounted on the top head arm to monitor the arm dynamics. A laser Doppler vibrometer (LDV) was used to detect the head-disk contact and monitor the transient head dynamics when a crash is happening. In this experiment, a typical Pemto slider $(1.235 \times 0.7 \times$ $0.23 \mathrm{~mm}^{3}$ ) covered by a $2 \mathrm{~nm}$ diamond-like carbon (DLC) film was tested. The nominal flying attitude was $10 \mathrm{~nm}$. The tested disk was a carbon coated 3.5-inch disk platter with a rotation speed of $5400 \mathrm{rpm}$. Signals were collected by an oscilloscope and a spectrum analyzer. The disk damage was inspected by an optical surface analyzer (OSA).

Figure 8 shows the signals monitored during the whole seeking process from inner diameter (ID) to outer diameter (OD) and then to ID. As observed from this figure, at phase III that the VCM current experienced, a vigorous vibration that occurred after the maximum back EMF was just generated. This vigorous vibration indicates a crash happening. Due to 


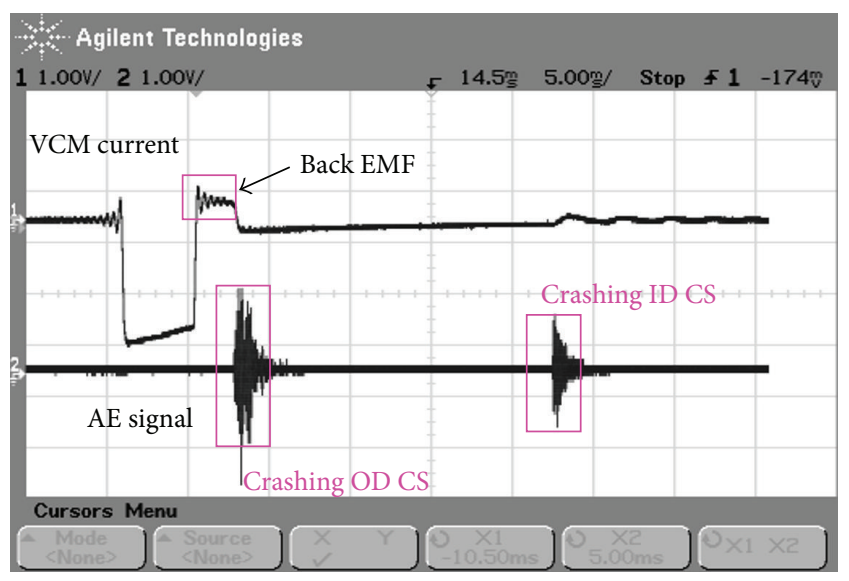

FIGURE 8: The monitored signals during seeking from inner diameter (ID) to outer diameter (OD), and then to ID. CS is the abbreviation of crash stop.

the crash, the seeking speed has a significant loss, leading to a sudden drop of the back EMF. By calculating the displacement that the head moved, this vigorous vibration can be identified as the crashing point between VCM end and OD crash stop. A very small response was observed at ID crash stop, indicating that the seeking speed has a significant loss after the crashing at OD crash stop.

\section{The Effect of Seeking Speed on HDD Instability}

4.1. Seeking Speed and Crashing Energy. The crash between VCM end and crash stop may induce a vigorous vibration of head and arm and further result in HDD instability such as head slap on disk and head/disk damage depending on the crashing energy. Seeking speed is the main cause of the crashing energy. To reveal the relationship between speed and crashing energy, crashing energy characterized by AE signal under different seeking speeds was investigated, as illustrated by Figure 9. It can be observed that, with the increase of seeking speed during crashing, the crash-induced vibration increases quickly.

To describe the crashing energy, the root mean squares (RMS) of AE signals were calculated under different crashing speeds. A clear increasing tread can be observed in Figure 10. From around $160 \mathrm{ips}$, the energy has begun to increase sharply, which indicated that HDD may become instable after this critical value. Further increasing the speed may lead to head/disk damage.

4.2. Head Dynamics. To monitor the head transient dynamics when crashing just happened, the laser spot of LDV was fixed on the position of OD crash stop to capture the vibration of head trailing edge center when head passed OD position. During the experiment, the laser will scan the trailing edge of head back when the head passed. Figure 11 shows the laser scanned area on the head back when head passed OD.
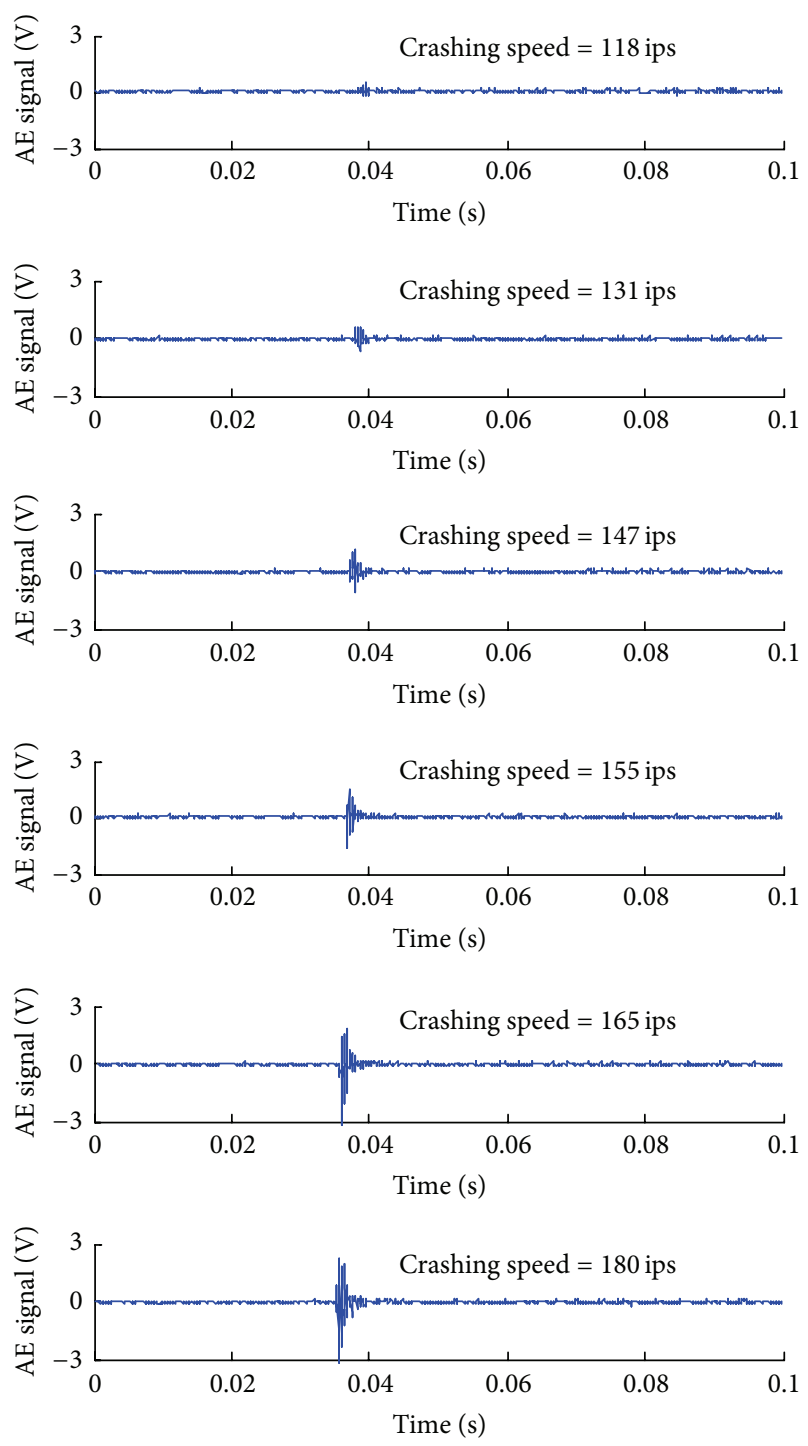

Figure 9: AE signals under different crashing speeds. ips is the abbreviation of inch per second.

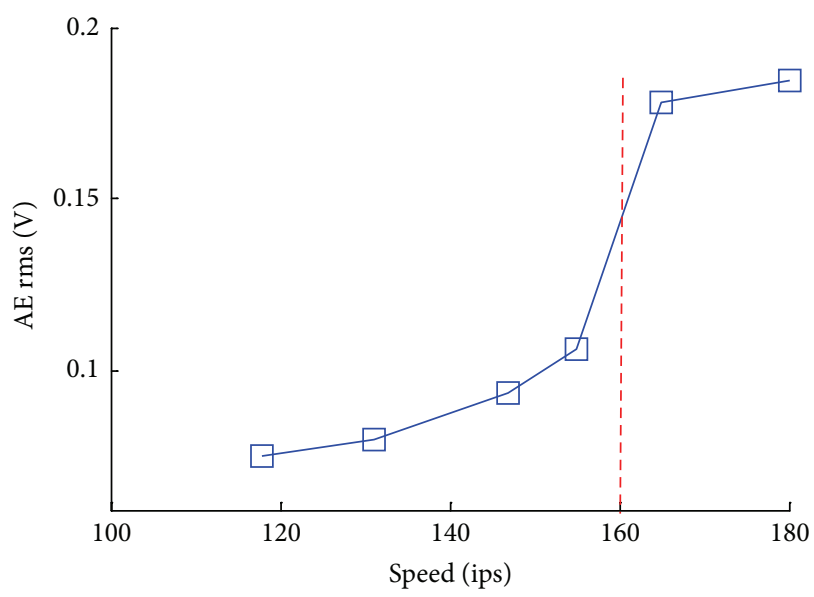

FIGURE 10: The energy generated by crashing under different seeking speeds. 


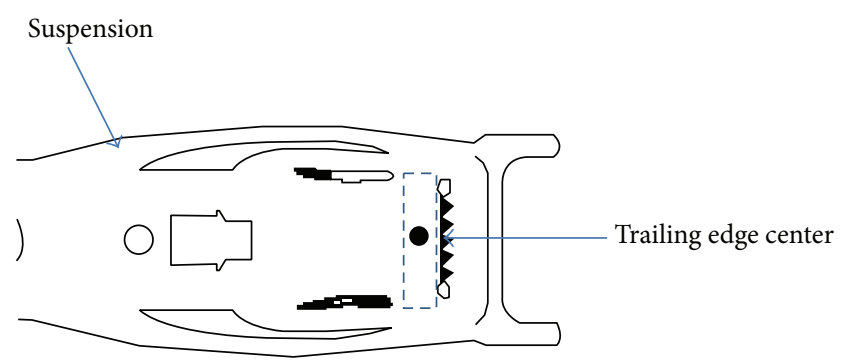

FIGURE 11: Laser scanned area on head when it passed to OD position.

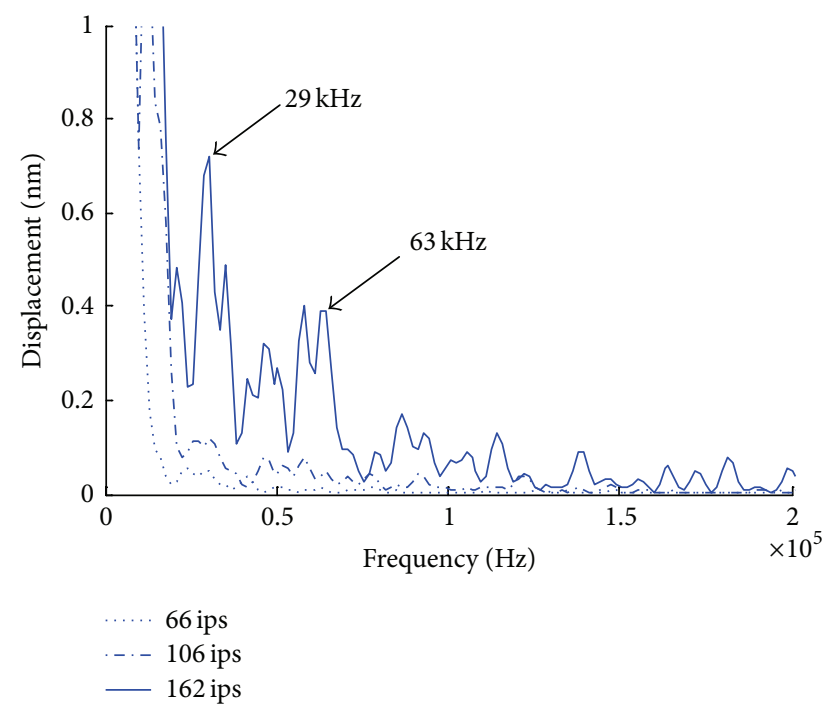

FIGURE 12: The frequency spectrum of LDV signal during crashing at $\mathrm{OD}$ crash stop.

A spectrum analyzer was used to acquire and analyze the laser signals with the cutoff of the frequency range of $10 \mathrm{kHz}-$ $200 \mathrm{kHz}$. Three different crashing speeds were conducted in order to reveal the relationship between the head dynamics and speed, as illustrated in Figure 12. As observed from this figure, when the crashing speed reached 162 ips, a large head vibration happened, while at 66 ips and 106 ips, there is nearly no significant response. This suggests that, at $162 \mathrm{ips}$, the air bearing may collapse and leads to HDI instability. The frequency spectrum of head response at 162 ips shows that the dominant modes are at $29 \mathrm{kHz}$ and $63 \mathrm{kHz}$. The responses of these two modes at 162 ips are about 10 times bigger than that of the other two speeds.

To reveal the dynamic nature of the head during crashing, a finite element model of whole head arm was built to identify the mode shapes of the two modes. The air bearing was modeled by four springs whose stiffness was identified by a parameter identification code [16]. The finite element model and the constraints are shown in Figure 13. Both modal and harmonic analysis were carried out using the finite element model. The mode shape of head under a lateral excitation (a unit force on coil end) showed that a torsion mode of head gimbal and the rolling mode of the air

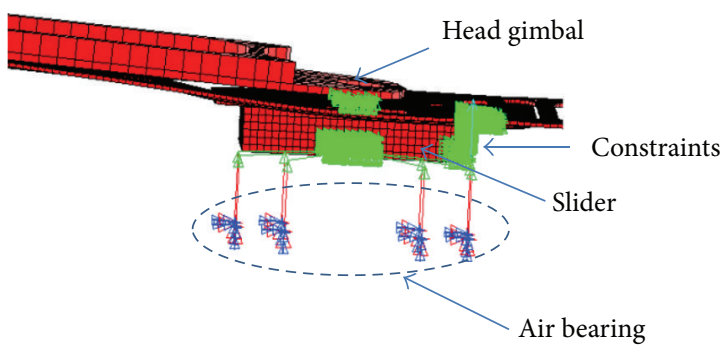

FIgURE 13: The finite element model of head arm.

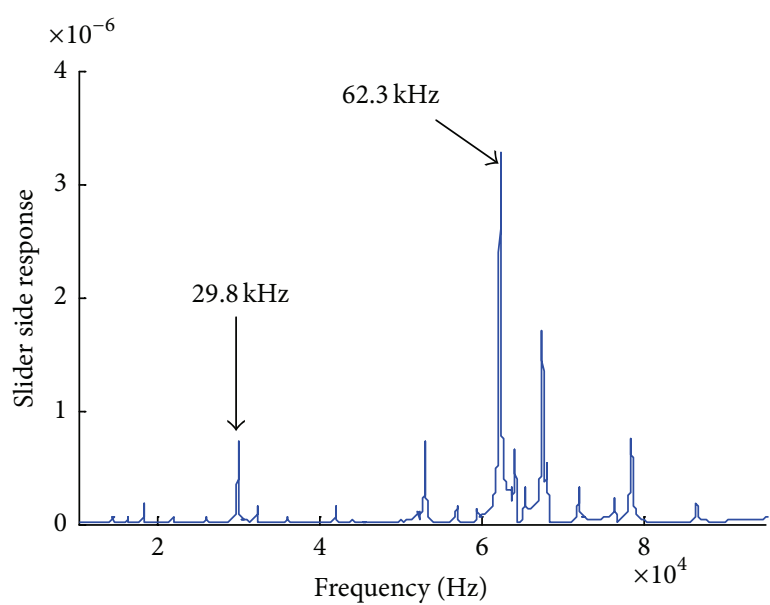

FIGURE 14: Head side response with a lateral excitation.

bearing dominated the head side response. The frequencies of these two modes $(29.8 \mathrm{kHz}$ and $62.3 \mathrm{kHz})$, as illustrated in Figure 14, are in accordance with the measured frequencies by LDV, respectively.

4.3. Damage Inspection of Disk Surface. After the experiment, the tested disks were inspected by OSA. Figure 15 shows the tested disk at crashing speed of 162 ips. As seen from this figure, some special patterns (damage) were observed, providing the evidence of air bearing collapse. The width of the patterns is about $0.7 \mathrm{~mm}$, which is equivalent to the head width, indicating that the head rolling motion dominates the head vibration when the arm end is crashing crash stop. This result is in good accordance with the mode shape that was identified by finite element model.

\section{Conclusion}

This paper investigated the effect of seeking speed on HDD instability. The head seeking was controlled by a back EMFbased technology. A mathematical method based on MLEEKF for deriving the seeking speed was developed. AE sensor and LDV were used to characterize the crashing energy and capture the head transient dynamics at the different speeds, respectively. With the help of finite element model and OSA inspection, the rolling mode of air bearing was found to dominate the head dynamics. This mode will lead to a serious damage of disk when a high seeking speed was adopted. 


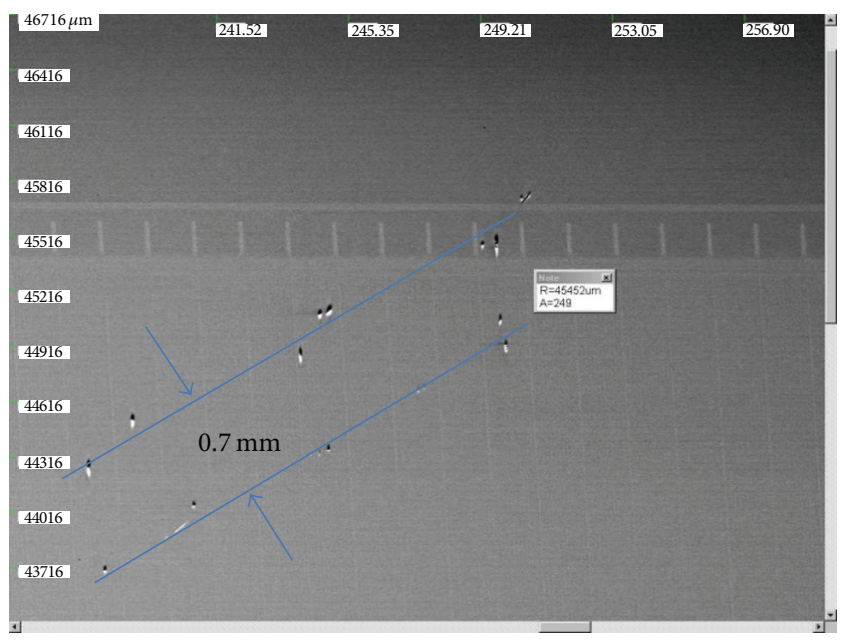

FIgURE 15: The OSA inspection result of disk after crashing test (crashing speed $=162 \mathrm{ips}$ ).

The results revealed in this paper will help to optimize the drive-level track seeking profile, as well as the design of components such as head and head arm.

\section{Conflict of Interests}

The authors declare that there is no conflict of interests regarding the publication of this paper.

\section{Acknowledgments}

This work was supported by the National Natural Science Foundation of China (51405380), China Postdoctoral Science Foundation (2014M560765), Hong Kong Innovation Technology Fund (GHX/003/12), and Hong Kong RGC GRF (121410).

\section{References}

[1] B. Marchon, T. Pitchford, Y.-T. Hsia, and S. Gangopadhyay, "The head-disk interface roadmap to an areal density of $4 \mathrm{Tbit} / \mathrm{in}^{2}$," Advances in Tribology, vol. 2013, Article ID 521086, 8 pages, 2013.

[2] V. Venkataramanan, G. Feng, and B. Gaber, "Track following servo control for rotary piezoelectric motor based primary actuation in hard disk drives," Microsystem Technologies, vol. 18, no. 9-10, pp. 1751-1757, 2012.

[3] B.-H. Chang and Y. Hori, "Trajectory design considering derivative of jerk for head-positioning of disk drive system with mechanical vibration," IEEE/ASME Transactions on Mechatronics, vol. 11, no. 3, pp. 273-279, 2006.

[4] D. Wu, G. Guo, and Y. Wang, "Reset integral-derivative control for HDD servo systems," IEEE Transactions on Control Systems Technology, vol. 15, no. 1, pp. 161-167, 2007.

[5] K. Peng, B. M. Chen, G. Cheng, and T. H. Lee, "Modeling and compensation of nonlinearities and friction in a micro hard disk drive servo system with nonlinear feedback control," IEEE Transactions on Control Systems Technology, vol. 13, no. 5, pp. 708-721, 2005.
[6] T. Yan and R. Lin, "Experimental modeling and compensation of pivot nonlinearity in hard disk drives," IEEE Transactions on Magnetics, vol. 39, no. 2, pp. 1064-1069, 2003.

[7] B. J. Harmer, "Closed loop calibration of back EMF measurement," US20100182715 A1, 2010.

[8] M. Yamashita, C. Ota, and N. Wasa, "HDD with VCM back EMF and voltage control circuitry," US patent 8497641 B2, 2013.

[9] R. Oboe, F. Marcassa, and G. Maiocchi, "Hard disk drive with voltage-driven voice coil motor and model-based control," IEEE Transactions on Magnetics, vol. 41, no. 2, pp. 784-790, 2005.

[10] K. Williams and T. Pham, "Rotating crash stop assembly for hard disk drives," US patent 5715119, 1998.

[11] R. E. Kalman, "A new approach to linear filtering and prediction problems," Transactions of the ASME-Journal of Basic Engineering, vol. 82, pp. 35-45, 1960.

[12] P. S. Maybeck, Stochastic Models, Estimation, and Control, vol. 141 of Mathematics in Science and Engineering, Academic Press, New York, NY, USA, 1979.

[13] J. C. Gamazo-Real, E. Vázquez-Sánchez, and J. Gómez-Gil, "Position and speed control of brushless dc motors using sensorless techniques and application trends," Sensors, vol. 10, no. 7, pp. 6901-6947, 2010.

[14] B. M. Chen, T. H. Lee, K. Peng, and V. Venkataramanan, Hard Disk Drive Servo Systems, Springer, London, UK, 2nd edition, 2006.

[15] P.-D. Pfister and Y. Perriard, "Very-high-speed slotless permanent-magnet motors: analytical modeling, optimization, design, and torque measurement methods," IEEE Transactions on Industrial Electronics, vol. 57, no. 1, pp. 296-303, 2010.

[16] Q. H. Zeng and D. B. Bogy, "Stiffness and damping evaluation of air bearing sliders and new designs with high damping," Journal of Tribology, vol. 121, no. 2, pp. 341-347, 1999. 

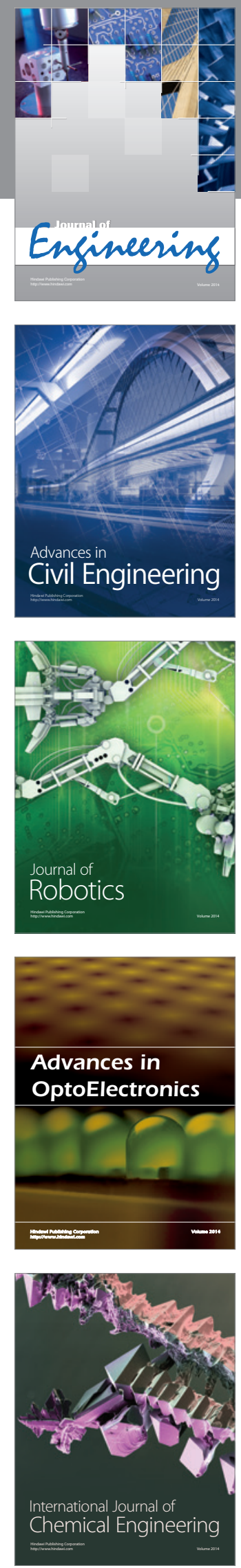

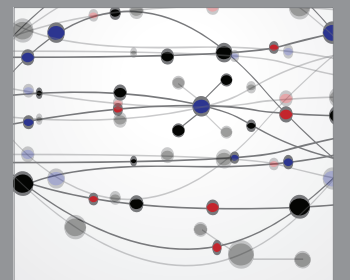

The Scientific World Journal
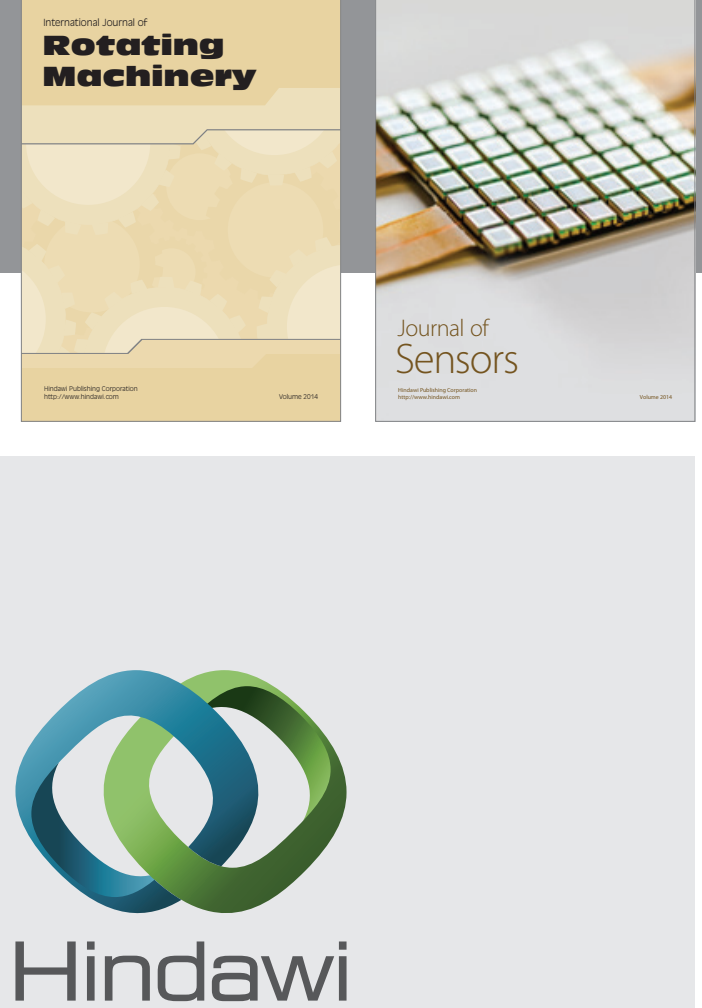

Submit your manuscripts at http://www.hindawi.com
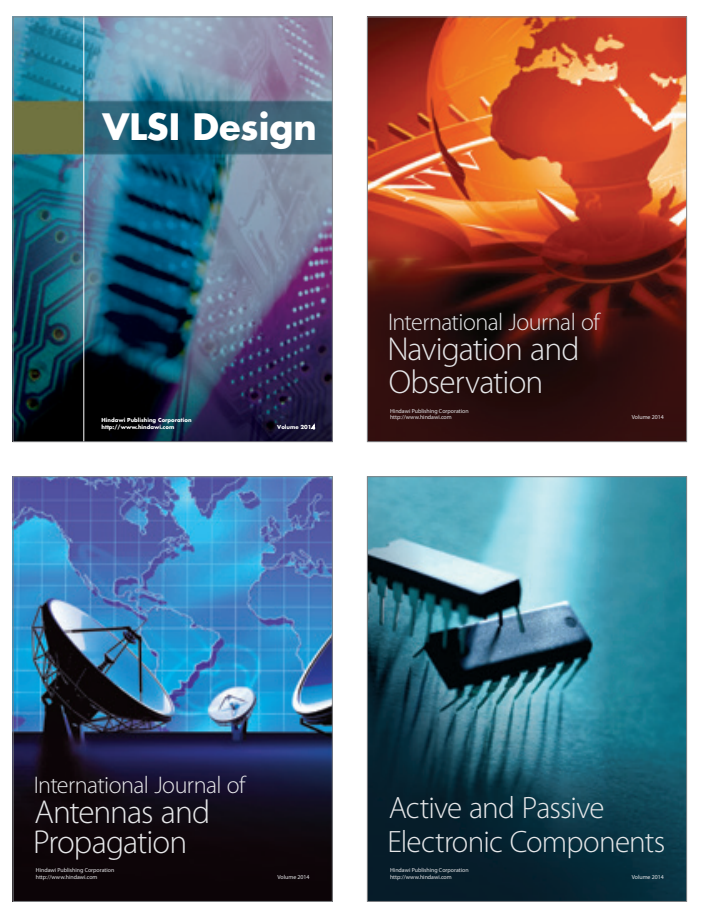
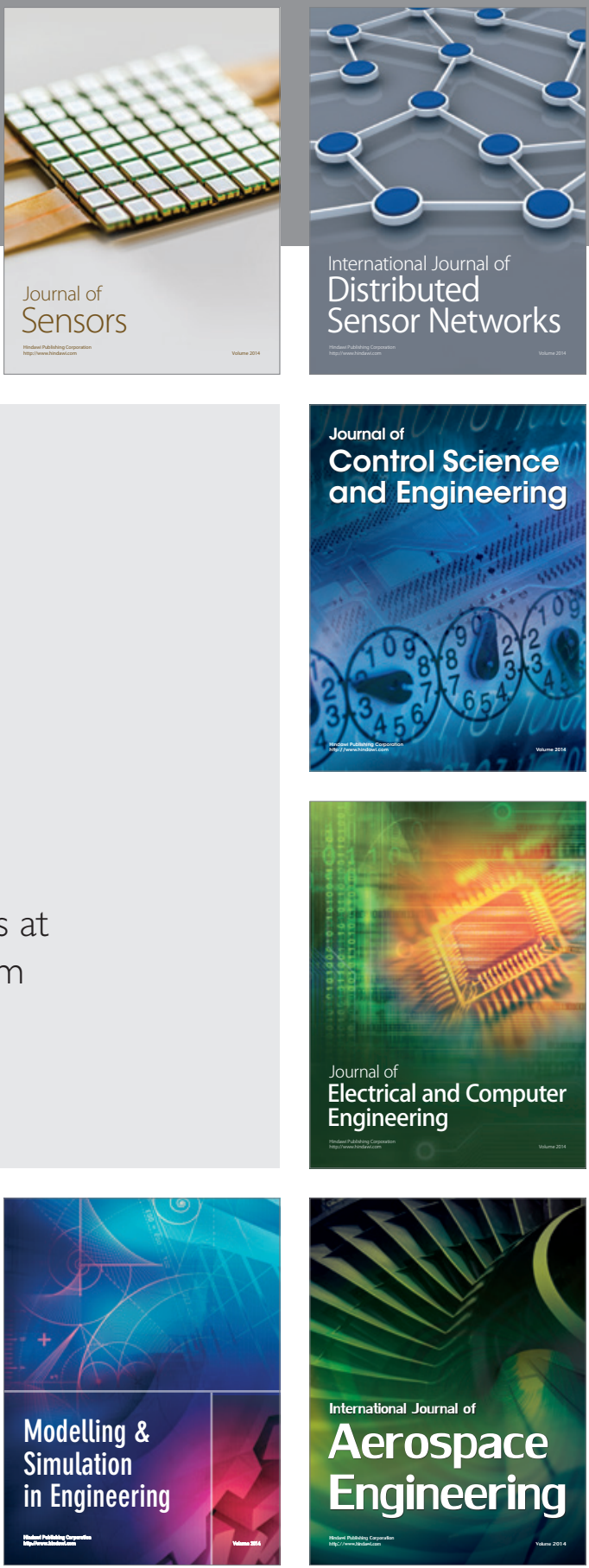

Journal of

Control Science

and Engineering
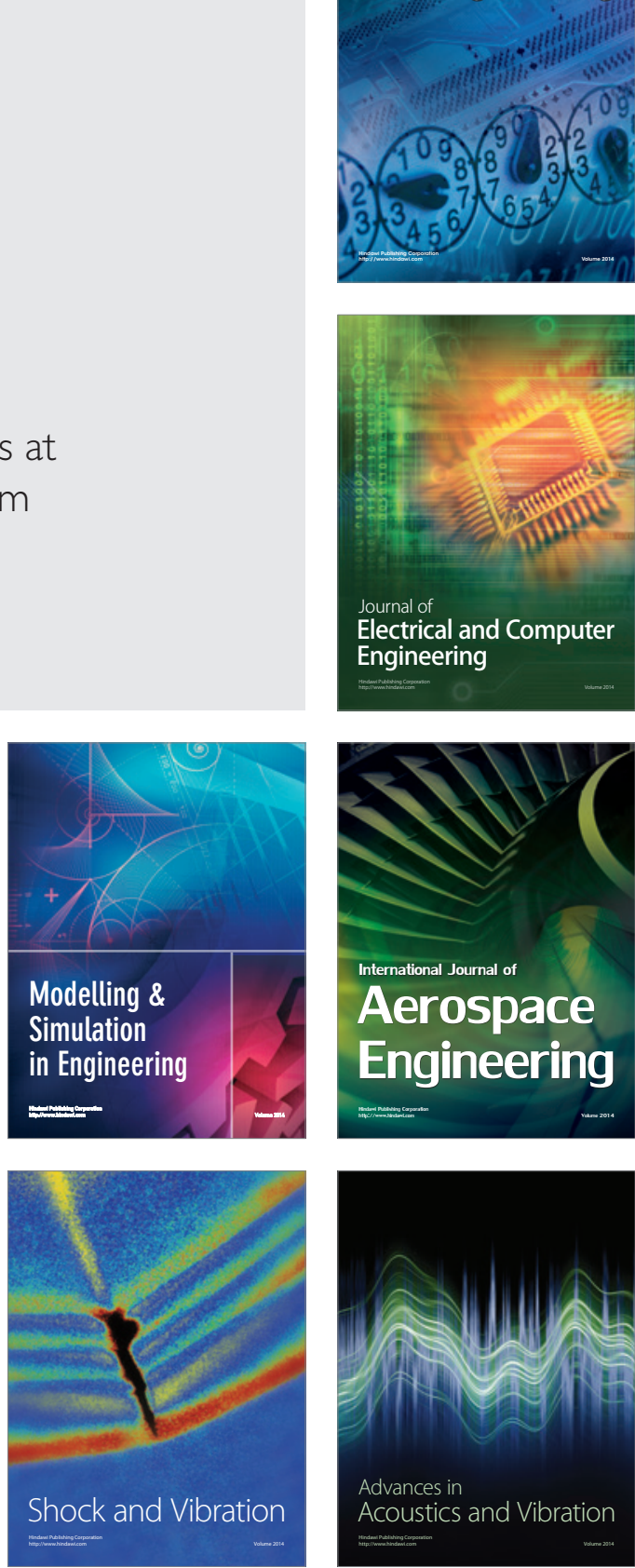